\title{
Vida e Historia: problemas sobre la relación entre la vida y la historia en Nietzsche.
}

\section{Life and History. Problems about the relationship between life and history in Nietzsche}

\author{
Daiana Marlén González \\ Estudiante de la Lic. y del Prof. en Filosofía \\ Universidad Nacional del Nordeste \\ marlengonzalez9679@gmail.com
}

\section{Resumen}

En la obra De la utilidad y los inconvenientes de la Historia para la vida, publicada en 1874, encontramos un planteo que tiene como uno de sus puntos principales el problema de la historia y su vinculación con la vida. En dicho escrito, la historia, como aspecto constitutivo de la vida humana, es centro de reflexiones y críticas. Partiendo de esta consideración, es decir, de una historia que tiene como motor profundas necesidades vitales y por ello, esencial para el desarrollo cultural, la relación de los hombres con su pasado es puesta en cuestión; de ello se desprende lo que -mencionado en su título- se reconocen como ventajas e inconvenientes para la vida. Desde una crítica a la historia erigida como ciencia pura analizaremos las tres formas en que la historia pertenece a la vida, es decir la historia monumental, anticuaria y crítica, sus utilidades y perjuicios.

Palabras claves: Historia - Vida - Nietzsche - Intempestiva - Ciencia

\begin{abstract}
In the work 'On the uses and Disadvantages of History for life' published in 1874, we discovered an approach which has as one of its central points the problem of history and its interrelation with life. In the work above-mentioned, the history, as a constituent aspect of the human life, is the focus of reflections and critics. As regards this consideration -about a history which has as boost deep vital necessities and therefore essential for the cultural development- the relation between the men with their past is controversial; from there it emerges what -mentioned in the title- is known as uses and disadvantages for life. Based on
\end{abstract}


a criticism to the history established as a pure science, we will be analyzing the three ways in which the history belongs to the life, which is the monumental, antique and critical history, their uses and detriments.

Key words: History - Life - Nietzsche - Untimely- Cience

\section{Introducción}

Para introducir al lector en el presente trabajo, mencionaremos algunos puntos concernientes a la obra en cuestión. Podemos decir que Nietzsche, cuando escribe sus Consideraciones Intempestivas se ubica en el rol de un crítico de la cultura a la que pertenece. En ese sentido, su intempestiva, será vista como un diagnóstico de la sociedad moderna, en especial de la sociedad alemana de su época. Posicionado en la función del filósofo-médico, que ya trascendió los trabajos exclusivamente ligados a la labor de filólogo clásico, Nietzsche lanza sus intempestivas sobre el problema de la historia y la necesidad de una historia para la vida. En su desarrollo, la principal crítica recaerá en lo que el autor considera una enfermedad de su época, el exceso de sentido histórico, dirá que "Tal vez, nuestra valoración de lo histórico no es más que un prejuicio occidental." (Nietzsche, 2002, p.27). Al mismo opondrá la necesidad de una fuerza plástica que se constituya no sólo a partir del recuerdo o la memoria, sino a partir del olvido, cuando éste sea necesario.

Antes de introducir al lector en el tema que nos convoca, será necesaria una última aclaración sobre la obra. Si hay que definir otro de los blancos a los que Nietzsche apunta en la intempestiva, aparecen como grandes figuras: el tan considerado historicismo y las filosofías de la historia. La modernidad, como bien mencionamos anteriormente, se caracteriza por exceso de sentido histórico, abundan en la época los estudios sobre culturas pasadas llegando al punto de una erudición que torna, tanto al historiador como a la historia mismas, inertes. En este sentido, a partir de una forma de hacer y entender la historia como una actividad científica no es precisamente el tipo de historia que habría que cultivar si lo que se busca el desarrollo de una cultura fuerte, este tiene que estar motivado por una fuerza mayor y diferente. Nietzsche (2002) lo expresa así: 
La historia concebida como ciencia pura, y aceptada como soberana, sería para la humanidad una especie de conclusión y ajuste de cuentas de la existencia. La cultura histórica es algo saludable y cargado de futuro tan solo al servicio de una nueva y potente corriente vital, de una civilización naciente, por ejemplo; es decir, solo cuando está dominada y dirigida por una fuerza superior, pero ella misma no es quien domina y dirige. (p. 28)

Es desde esta bifurcación, la de una historia que pretende erigirse como ciencia pura y la de una historia que tiene como motor la vida y está al servicio de ella que parte el presente trabajo. Nos centraremos aquí, en esta segunda dimensión interpretativa, en las formas que adopta la relación de los sujetos con su pasado, con su historia cuando esta es dirigida por las necesidades y los impulsos vitales; cuáles son en este aspecto, sus ventajas e inconvenientes para la vida. Para dicho propósito, analizaremos los tres tipos o modos de historia que para Nietzsche pertenecen a la vida ${ }^{1}$ y en consecuencia concluimos intentando responder si es posible para el autor un abordaje científico del devenir histórico, y en tal caso, qué consecuencias tiene este para la vida. Estas tres formas o modos de historia serán: la historia monumental, la historia anticuaria y la historia crítica.

\section{Lo intempestivo, el sentido histórico y la fuerza plástica}

Nietzsche se encarga, en este escrito que llegó a considerar belicoso, por decirlo en sus propias palabras, de definir y plantear el problema del valor y el no valor que la historia tiene para la vida humana. Por ello se entiende el porqué lo considera un escrito intempestivo. El autor expresa que llega a tal experiencia intempestiva a partir del aprendizaje de culturas pasadas y, especialmente, a través de una manera particular de entender la cultura griega. Dicho esto, se encarga de esclarecer el término intempestivo, esto último, agrega Nietzsche, tiene que ver con una forma de interpretación y esta se caracteriza porque ve como un mal, una enfermedad o un defecto un objeto de orgullo para una época (Nietzsche, 2002, p. 19). Ese objeto de orgullo de la modernidad alemana, es ni

\footnotetext{
${ }^{1}$ El sentido de esta frase: una historia que pertenece a la vida, será aclarado en el siguiente capítulo por ser de suma relevancia para el presente estudio.
} 
más ni menos que su tan valorado sentido histórico, su cultura historicista. Primera transvaloración de Nietzsche, la consideración de un valor época como un signo de la decadencia moderna.

Por lo mismo señala, y nuevamente resuenan las disputan con los filólogos clásicos de su tiempo, que la filología si tiene algún deber con respecto a su estudio este debe ser el poder actuar, contra el tiempo, sobre el tiempo en favor y beneficio de un tiempo nuevo.

Es importante recordar que Nietzsche, como mencionamos al comienzo, tiene la intención de establecer el valor o no valor de la historia en pos de la vida. Esto puede interpretarse como la primera aproximación a la interpretación del I valor de la historia a partir de las consecuencias para la acción, sea que la potencie, que vaya en búsqueda de las grandes acciones o que la aniquile y degenere. En palabras del filósofo:

(...)la historia, en cuanto preciosa superfluidad del conocimiento y artículo de lujo, nos han de resultar seriamente odiosas, según la expresión de Goethe precisamente porque nos falta lo más necesario y lo superfluo es enemigo de lo necesario. Es cierto que necesitamos la historia, pero de otra manera que el refinado paseante por el jardín de la ciencia, por más que este mire con altanero desdén nuestras necesidades y apremios rudos y simples. Es decir, necesitamos la historia para la vida y la acción ${ }^{2}$, no para apartarnos cómodamente de la vida y la acción (...) Tan sólo en cuanto la historia está al servicio de la vida, queremos servir a la historia. (Nietzsche, 2002, p. 18)

A diferencia de una historia al servicio de la vida, se encuentra un tipo de historia que muy vinculados a las ciencias de la época, intenta fundarse como tal. Prevalecen en esta corriente los criterios de validación científica legalistas, objetivos y predictivos.

La concepción de Nietzsche acerca de la historia, debe contextualizarse en una época profundamente historicista, el siglo XIX; aun cuando reinaba el positivismo, los historiadores de la época, en su afán por reivindicar la categoría epistemológica de la Historia como Ciencia, originaron una corriente que perdurará hasta los inicios del siglo XX: tal corriente se denominará historicismo, entendida como una

\footnotetext{
${ }^{2}$ La cursiva no pertenece al escrito original, es un agregado de este trabajo.
} 
afirmación de que la vida y la realidad están determinadas históricamente y de que todo hecho histórico debe ser entendido desde su «propio» y «aparente» ser. (Correa Gutiérrez, 2008, p.66)

Existe una convicción en esta forma de hacer de la historia una ciencia, tanto en el progreso de la misma y en la posibilidad de sustancializar la historia, como de encontrar sus determinaciones objetivas que se sostiene en parte en la creencia en un tiempo histórico lineal, progresivo y que tiene sus orígenes en una concepción cristiana de la historia. Esto tiene como consecuencia, una excesiva valoración del conocimiento histórico, que desde la perspectiva nietzscheana además de ser un engaño, aunque a veces útil, degenera y debilita la vida, ese mismo exceso de valoración del conocimiento y la idea de verdad que ya había sido denunciado en Verdad y Mentira en sentido extramoral.

La objetividad pretendida por el historicismo y el positivismo de la época no es posible. Frey (2015) dirá que:

En su crítica al historicismo de corte rankiano, Nietzsche tiene claro que el historiador es el que debe conferirle al pasado una estructura de significado para construir con ella una historia en el sentido de una narrativa. Esto significa que de un mar inagotable de acontecimientos isotrópicos sólo selecciona un número finito; algunos los patetiza, otros los memoriza y otros más los olvida — pero, en realidad, a la mayoría ni siquiera los toma en cuenta. (p. 276)

En esta forma intempestiva de considerar el exceso del sentido histórico, o mejor dicho, la valoración de la historicismo de la época, no sólo la objetividad y legalidad no son posibles, sino que además debilitan y degeneran los instintos más fuertes de una cultura “(...) hay un grado de insomnio, de rumiar, de sentido histórico, en el que lo vivo se resiente $y$, finalmente, sucumbe, ya se trate de un individuo, de un pueblo, o de una cultura." (Nietzsche, 2006, p. 22) Lo que el autor nos intenta mostrar es que en la tensión que se erige a partir del exceso de sentido histórico, o, lo que es igual, en la desmedida consideración de pasado es la vida misma quien corre peligro. La amenaza del exceso de historia para la vida, será entonces una nota de gran importancia en esta crítica. Este cultivo hipertrófico del conocimiento histórico, prohíbe la generación de la historia misma, "Un 
fenómeno histórico, pura y completamente conocido, reducido a fenómeno cognoscitivo es, para el que así lo ha estudiado, algo muerto (...)" (Nietzsche, 2006, p. 28)

En consecuencia, historia y vida estarán en constante relación y tensión según prevalezcan los instintos más nobles o los más bajos, según la fortaleza o debilidad del carácter de una cultura o individuo que dan lugar a una relación sana o enfermiza con el pasado.

Partiendo de la idea de que es más importante para la vida y la felicidad humana más que la capacidad de recordar- la fuerza para olvidar, el autor traza una comparación muy interesante entre los animales y los hombres. En esta comparación, es el animal quien vive en constante estado de felicidad, pues no sabe recordar, el presente es lo único constante y por ello, la vida se desarrolla sin el peso del pasado. En cambio, el hombre que vive atado a su pasado tiene a este como su principal cadena, Nietzsche nos invita a imaginar la existencia de un individuo que no pudiera olvidar nada, la consecuencia lógica para él sería la parálisis absoluta, la imposibilidad de acción. De acuerdo con lo dicho, el hombre se ubicaría entre el animal que todo lo olvida y la hipotética figura de ese hombre que recuerda cada instante de su vida. Es posible entonces, para el hombre, aprender a olvidar y no solo es posible sino que es necesario en tanto que sea ávido de grandes acciones y desee la felicidad, pues no es feliz para Nietzsche quien no es capaz de olvidar y lo que es aún peor en su consideración: no puede hacer feliz a nadie a su alrededor quien es incapaz de adentrarse en la fuerza del presente, en el umbral del momento por pensar en lo que ya ha acontecido. A esta capacidad de olvidar la llamaremos sentido ahistórico o capacidad de sentir ahistóricamente. Para la fecundidad de una cultura es necesario entender cuándo se requiere "sentir las cosas desde el punto de vista histórico o desde el punto de vista ahistórico. Lo histórico y lo ahistórico son igualmente necesarios para la salud de los individuos, de los pueblos y de las culturas." (Nietzsche, 2002, p.23).

A esta altura, resulta evidente, la contraposición con el modo historicista de considerar la historia fundada en una erudición que torna inerte a los estudios históricos. En estas formas de considerar la historia al servicio de la vida, el olvido se constituye como una acción indispensable precisamente, para la actuación. Aquí es donde emerge la pregunta sobre qué es lo que debiera olvidarse, teniendo en cuenta nuestra interpretación del texto nietzscheano, diremos que aquello que se debe olvidar esta vinculado con lo que no se puede dominar, lo que torna a la vida débil o lo que paraliza la acción. Si bien estas 
consideraciones no se pueden tomar literalmente como prescripciones universales, una mención a los que Nietzsche considera indispensable podrá servir de guía para la interpretación. Es necesaria entonces, lo que Nietzsche (2002) denomina fuerza plástica:

(...) habría que saber con exactitud cuánta es la fuerza plástica de un individuo, de un pueblo, de una cultura. Me refiero a esa fuerza para crecer desde la propia esencia, transformar y asimilar lo que es pasado y extraño, cicatrizar las heridas, reparar las pérdidas, rehacer las formas destruidas. (p.22)

\section{Tres formas de relación con la historia: monumental, anticuaria y crítica}

Lo primero que diremos, es que de la salud de un pueblo depende en gran medida de responder hasta qué punto es necesaria la historia, el recuerdo de su pasado. En otras palabras es necesario definir en qué medida sirve la historia a la vida, a la salud, a la fecundidad y a la fuerza del presente y al servicio del porvenir. Volviendo al problema que vincula el exceso de sentido histórico a la enfermedad de su tiempo, Nietzsche que atiende especialmente a la cuestión de la ciencia de la historia y sus consecuencias para la cultura, una vez hecha la crítica, se centrará en brindar tres formas de hacer y entender la historia, es decir tres modos en que es posible relacionarse con el pasado que no pertenecen al ámbito de la historia como conocimiento puro y objetivo sino que responden exclusivamente a necesidades vitales.

A esto hicimos mención más arriba cuando mencionamos la necesidad de una aclaración del término "pertenecen a la vida". Esta tres formas en que la historia pertenece a la vida tienen que ver precisamente con cuál es la fuerza que la dirige. Es inevitable que surja una duda con respecto a la ciencia histórica, cuál es su surgimiento, a qué necesidades responde. También detrás de ella se encuentra la cuestión de la vida y sus impulsos pero estos están dirigidos y orientadas por una voluntad de verdad y de conocimiento. Diremos en cambio, que en la historia monumental, anticuaria y crítica esta pertenencia se 
corresponde con tres tipos de necesidades vitales ${ }^{3}:$ “(..) en la medida que es un ser activo y persigue un objetivo, en la medida que preserva y venera lo que ha hecho, en la medida que sufre y tiene necesidad de una liberación." (Nietzsche, 2002, p.28). Pasemos a considerar estos tres modos.

El primero de ellos lo denominamos historia monumental ¿Que hay detrás de un pasado monumental? ¿Qué individuos, instintos y necesidades se esconden bajo esta forma de considerar el pasado? ¿Cuáles son sus consecuencias para la vida? Nietzsche considera que los dueños y los grandes hombres de la historia son aquellos dotados de grandeza, de esa gran fuerza plástica necesaria para apropiarse del pasado y servirse de él. Estos hombres son los capaces de desatar grandes luchas y lo hacen con el afán de gloria e inmortalidad. La necesidad que se esconde detrás de estos individuos es la de lograr grandes cosas, ser productores de grandes acciones y acontecimientos. Por lo mismo, la única historia capaz de servirle es aquella que contenga los grandes relatos de la humanidad, sus mejores figuras y sus mayores hazañas.

La cuestión se formula aquí a partir de la pregunta por el quién necesita de una historia monumental. Este quién, se configura detrás de los mayores personajes históricos de la humanidad. Estos hombres al experimentar la imposibilidad de encontrar en su tiempo presente los ejemplos de los cuales mirarse a sí mismos y guiarse en la acción, se sirven de la historia y buscan en su pasado modelos de los cuales apropiarse y maestros de los cuales aprender. La historia que se dan es por lo tanto, aquella que contenga la grandeza suficiente que les permita creer que ellos son igualmente capaces de producirlas. Existe aquí un deseo de gloria y también la creencia de que la humanidad puede alcanzar nuevos hitos, hay fe implícita en el progreso de la historia y a su vez, la idea de que de la historia es posible aprender. Nietzsche (2002) dirá que el individuo de la historia monumental:

“Deduce que la grandeza que un día existió fue, en todo caso, una vez posible, y, sin duda, podrá, otra segunda vez, ser posible; anda su camino con paso más firme, pues la duda que le asaltaba en momentos de debilidad, de si estaría aspirando tal vez a lo imposible, se ha desvanecido" (p.31.)

\footnotetext{
${ }^{3}$ Cragnolini (2009) señala que: “(...) el viviente necesita de este servicio de la historia, y lo necesita desde tres necesidades bien diferenciadas: como un ser que actúa, como un ser que venera, y como un ser sufriente que anhela liberación" (p.143).
} 
En este sentido, se entiende cómo de esta forma de relacionarse con el pasado, la vida del individuo resulta potenciada y fortalecida. La historia le muestra que aquellos grandes acontecimientos que espera realizar, aquello que anhela fue posible y puede serlo una próxima vez. Decae la debilidad e inseguridad en torno a sus aspiraciones, y por lo tanto, la vida misma es beneficiada, acrecentada en su fuerza para la acción.

Ahora bien ¿Qué sucede cuando no es solo el individuo de acción y poderoso quien tiene necesidad de esta historia? ¿Qué pasaría si quien se sirve de este tipo de historia es el individuo pasivo e impotente? Nietzsche señala que además del individuo activo y potente, también tiene necesidad de este tipo de historia aquel quien no es capaz de grandes cosas, quien no posee el carácter y la fuerza suficiente para las grandes acciones. Pero como se verá, en este caso, los instintos que imperan son otros. Es la impotencia, los celos y el resentimiento los que fundamentan este tipo de historia. Estos glorifican y veneran una historia monumental solo como un instrumento, como un disfraz para su resentimiento hacia las mejores figuras de su presente. Lo que el autor señala es que el individuo impotente es incapaz de reconocer en sus semejantes a aquellos que están dispuestos a escribir la historia, por lo mismo se da un pasado monumental a fin de menospreciar a los mejores individuos de su época. Una excesiva consideración del pasado, en desprestigio de las grandes acciones y los caracteres más fuertes del presente, da cuenta de la utilización del pasado de un espíritu del resentimiento. En esta forma de apropiación la historia, la vida se ve perjudicada.

Ubicándonos en el segundo modo en que la historia le pertenece a la vida, nos encontramos con la historia anticuaria. Para Nietzsche, esta forma es propia de quien mira a su pasado con veneración y amor. Podemos decir que el principal servicio que brinda este tipo de relación con el pasado es el de la preservación para su posteridad. Esta preservación parte de un deseo y necesidad de sentirse heredero de un pasado. Caracterizada por un sentido de pertenencia, le da a un pueblo o a un individuo un pasado común del cual proceder y hace que se olvide de la inseguridad y arbitrariedad propias de la existencia. Existe por lo tanto, una suerte de justificación de la existencia presente a partir del pasado común, de la herencia, de la procedencia, ya sea de un pueblo, una cultura, una tradición, etc. Esto último es una de las principales utilidades de la historia anticuaria. 
Además de esto, el autor menciona las principales características de quienes se sirven de esta historia; ellas son la amplitud de su sensibilidad, de su intuición y capacidad de presagio. A modo de ilustración, Nietzsche dirá que todas esas cualidades son las mismas que guiaron a los italianos del Renacimiento en sus creaciones culturales, que despertaron en ellos el genio ático. El sentido histórico se encuentra particularmente representado aquí por individuos de tal naturaleza.

Ahora bien, el problema comienza cuando dichas virtudes se petrifican y ya no sirven a la vida conservandola sino que produce la momificación del pasado y del presente. Podemos decir que uno de los principales peligros de los instintos que dan lugar a la historia anticuaria es su excesiva fuerza. La necesidad de conservación y la sobredimensión de la veneración del pasado suelen alcanzar una fuerza tal que absorben y eliminan a las demás formas de servirse de la historia y de relacionarse con el pasado. Es en este escenario, donde los instintos de conservación llegan a tal punto de generar lo que Nietzsche se inclina a llamar una ciega furia coleccionista.

Ya sin la mirada fiel y amorosa sobre el pasado, la historia anticuaria se convierte en una autosatisfacción erudita de acumulación de conocimientos históricos y objetos pretéritos. Lo que tiene como consecuencia que las otras necesidades, más orientadas a la valoración del presente y el futuro, no encuentran modo fecundo de ser satisfechas. Por ello, "La historia anticuaria degenera, en el momento que ya no está animada e inspirada por la fresca vida presente. Entonces la piedad se marchita..." (Nietzsche, 2002, p.) a lo que se concluye que "La historia anticuaria sabe solo cómo conservar la vida, no cómo crearla" (Nietzsche, 2002, p.38). Esto se entiende mejor, si se piensa cuán difícil es la consideración positiva acerca de sucesos novedosos, o formas de vida que intenten instaurar nuevas costumbres para quien ve en el pasado aquello de más valor. Toda novedad representa para el individuo anticuario una amenaza a la tradición, a lo institucionalizado y de alguna manera naturalizado.

En constante disputa con esta clase de historia se encuentra lo que llamaremos historia crítica, de la cual Nietzsche (2002) dirá que:

Para poder vivir, ha de tener la fuerza, y de vez en cuando utilizarla, de romper y disolver una parte de su pasado: esto lo logra trayendo ese pasado ante la justicia, sometiendolo a un interrogatorio minucioso y, al fin, condenando; todo pasado 
merece condenación, pues tal es la naturaleza de las cosas humanas: siempre la humana violencia y debilidad han jugado un papel importante. (p. 38)

En esta forma violenta de relación con el pasado, lo que hay son individuos, pueblos y culturas enteras que sufren con su presente. Al considerar al presente como producto de de un pasado común, se reconoce que éste es el fruto de los errores de la historia y si el presente genera padecimiento hay que atacar en primer lugar su causa, a aquello que le dio lugar, su historia. La necesidad de condenación, de juicio de las acciones pasadas como principal causante de los dolores actuales es lo que da lugar a una memoria crítica. La cuestión de la tensión entre memoria y olvido vuelve a aparecer; y si bien el autor consideraba la capacidad de olvidar como indispensable para la salud de un pueblo o individuo, lo que se hace necesario ahora es el recuerdo. La actualización del pasado en el presente no estará vinculada con una interpretación fiel del pasado ni de veneración de su historia, es más bien una necesidad de juicio y condena de una historia considerada hostil. Esta relación con el pasado no está alejada de peligros y riesgos para la vida.

Para entender esto último es conveniente primero responder a la pregunta sobre quién está a cargo de condenar el pasado, esta es afirmará Nietzsche (2002) la vida misma “(...) esa potencia oscura, impulsiva, insaciablemente ávida de sí misma. Su veredicto es siempre inclemente, siempre injusto (...)" (p. 38) El riesgo se da porque en el intento de destrucción y condena del pasado se ignora hasta qué punto la propia naturaleza está constituida por él. El pasado es una cadena y un peso del cual es muy difícil librarse sin destruir al mismo tiempo lo más fuerte y fecundo que hay de la naturaleza presente. Es por lo tanto, más que la historia, la vida misma la que está en constante riesgo.

Ante este escenario, se pueden dar dos situaciones. En el caso más extremo, Nietzsche señala que puede producirse una completa antagonía entre el pasado propio y las concepciones presentes, estos serían en tal situación inconciliables. La otra alternativa supone el escenario para entablar una lucha con ese pasado, mediante la cual "(...) cultivamos un nuevo hábito, un nuevo instinto, una segunda naturaleza, de forma que la primera desaparezca." (Nietzsche, 2002, p. 39). Pero ¿cómo se llega a esa sustitución, a ese cambio de naturaleza? Mediante un disciplinamiento, una nueva rutina y hábitos de gran rigurosidad. En esta contienda, está siempre la posibilidad de perder, pues el autor considera que uno de los elementos a tener en cuenta es que las segundas naturalezas son 
en sus estados de gestación más débiles que las primeras. Hay un riesgo manifiesto de que el intento de darse una nueva naturaleza genere un estado vital mucho más débil e impotente que aquel que se pretende destruir. Independientemente de sus posibilidades negativas, eso no hace menos necesaria a la historia crítica. El autor considera que más allá de esto, el consuelo de quien se apropia críticamente de la historia es la conciencia de que las primeras naturalezas han sido en algún momento las segundas y que, si logran triunfar, se constituirán en las primeras para la posteridad.

Por último, aquí se abre también la posibilidad de pensar en la repetición de esta operación. Ante la imposibilidad de separar el hecho mismo de la existencia de la injusticia de la misma. Siempre un estado de cosas, un orden, jerarquía, etc, resultan injustos para alguien; así también la constitución de una segunda naturaleza que va en contra de la injusticia de la primera será en un futuro considerada injusta y llevada a juicio.

Hasta aquí lo analizado con respecto a las vinculaciones existentes entre historia monumental, anticuaria y crítica en su vinculación a las necesidades vitales que la fundamentan. Para cerrar nos retimitos a las palabras de Nietzsche (2002):

Estos son los servicios que la historia puede prestar a la vida. Todo individuo, todo pueblo necesita, según sus objetivos, fuerzas y necesidades, un cierto conocimiento del pasado, ya sea como historia monumental, anticuaria o crítica. Pero no como lo necesitaría un tropel de puros pensadores que no hacen más que asistir como espectadores a la vida, o individuos sedientos de saber, que solo con el saber se sienten satisfechos y para quienes el aumento de conocimientos es el objetivo en sí, sino, siempre y únicamente, con vistas a la vida y, por tanto, bajo el dominio y suprema dirección de la misma. (p. 40)

\section{Algunas consideraciones finales sobre la relación entre vida e historia}

Para finalizar nos encargaremos particularmente de mencionar y comentar algunos de los problemas y consecuencias que se generan a partir de la propuesta nietzscheana de vincular la historia a un aspecto vital, instintivo e interesado para la pretensión de la ciencia 
histórica en su pretensión de objetividad. Para lo mismo tomaremos elementos de cada uno de los modos de historia descriptos que estén vinculados directamente con el aspecto gnoseológico de lo histórico. Tomaremos entonces de la historia monumental los elementos de repetición, de comparación y homologación de los hechos históricos. Con respecto a la historia anticuaria tomaremos sus criterios de jerarquización y valoración. Y por último, en cuanto a la historia crítica nos encargaremos de su falta de justicia y el corrimiento de una tesis arraigada en la época, vinculada a Hegel de pensar que es la historia quien juzga y no la vida y los instintos humanos.

Tal vez, desde la propuesta de Nietzsche no haya un modo de historia en la que el conocimiento histórico sufra tanto como en el caso de la historia monumental. En la necesidad que vimos de un aprendizaje, de maestros, de ejemplos de los cuales la historia nos proveería, hay como ya dijimos una idea de que es posible que los grandes hechos pueden repetirse y que es posible servirse de la historia como lugar para la justificación de grandes acciones. Es necesaria entonces una comparación y homologación de hechos. Si lo que es grande puede suceder otra vez, lo que subsiste es la creencia de que las causas que lo generaron pueden volver a darse. Para Nietzsche esto es imposible en los hechos, lo que sucede entonces es que el relato o el conocimiento histórico sufren recortes, arbitrariedades, grandes fragmentos de la historia son olvidados. Solo a partir de estos procedimientos es posible dotar de tal grandeza a algunos hechos o personajes históricos hasta el punto de la mitificación. Se da lugar a lo que el autor llama una serie de efectos en sí, es decir, el punto de vista que ve en la historia grandes hechos en deterioro del olvido de gran parte de causas y fundamentos, que generaría en manos de algunos la creencia de que es posible una determinada cantidad de hechos sin causas suficientes. Nietzsche considera que mientras la historiografía siga en gran medida influenciada por la historia monumental se parecerá en muchos aspectos a un relato mitológico y hasta poético, pues para la historia monumental la veracidad no es el elemento principal.

Vista desde el ámbito de la historia anticuaria, es tal vez la vida lo que más se encuentre degenerado, no por el riesgo que corre sino por la excesiva pasividad que la convierte en la principal enemiga de toda acción nueva en gestación. En cuanto a la relación con la historia en términos de conocimiento y valoración esta tiene por principal defecto el carecer de una jerarquía de valores. Mientras Nietzsche afirma la importancia del olvido, 
propio de una atmósfera fecunda para el nacimiento de grandes, fecundas y fuertes acciones, en la historia anticuaria existe una falta de medida o valor que permita poner de un lado aquello que merece ser recordado y lo que no. Son estas las condiciones que dan lugar a la mencionada furia coleccionista del anticuario. Para quien todo hecho pasado es igualmente digno de ser recordado y venerado, cualquier acción presente que valore el pasado de igual manera representa una profanación a su propia historia. Radica aquí una crítica a la desmedida erudición, pues tiene al conocimiento del pasado como fin en sí mismo, no como beneficioso y potenciador de acciones presentes, sino que sofoca y mata todo impulso vital nuevo. La historia se ve afectada al convertirse en un conocimiento inerte.

Finalmente, cuando hablamos de historia crítica vemos en Nietzsche un desplazamiento de un elemento muy valorado en la ciencia de la historia, el deseo de hacer justicia con el pasado, de contar los hechos tal y como sucedieron verdaderamente. En la mención que hace el autor sobre quién es el juez en la historia crítica Nietzsche dirá que es la vida quien juzga y no la historia. Vida e injusticia son dos aspectos que van de la mano, pues “(...) se requiere mucha fuerza para poder vivir y olvidar que vivir y ser injusto son la misma cosa." (Nietzsche, 2002, p.39). Cuando la mayor parte de la fuerza de la historia crítica se debe a su capacidad de juzgar un pasado y condenarlo, si esta condena procede de la vida misma, su sentencia nunca puede ser justa. No es aquí la fuente de conocimientos históricos objetivos quienes establecen los criterios justos para la sentencia, se juzga al pasado por el hecho de haber sido y de formar parte aún de un presente que causa sufrimiento. Que los hechos hayan ocurrido de tal o cual manera importa poco a la historia crítica, se los juzga por haber sucedido y por sus consecuencias, de ahí que la crítica que destruye y da muerte a su pasado, sea la que abre el camino a las segundas naturalezas, a las luchas entre el pasado y futuro, a la vida en su generación. Por lo tanto, este tipo de historia nunca puede cumplir con el parámetro de justicia para con el pasado, ni expresar los hechos tal y como fueron como es pretendido por la historia como ciencia. 


\section{Conclusiones}

Hemos realizado un recorrido que pretendía servir de introducción al texto de Nietzsche "De la utilidad y los inconvenientes de la Historia para la vida". A partir del mismo se llegó a poner como centro del planteo la vinculación, clave para el autor, entre vida e historia. Partiendo de una crítica cultural se hizo patente el interés de Nietzsche por cuestionar el estatus que gozaba la ciencia histórica de la época, dominada por deseo de determinación de la vida a partir de la historia. Así como también, se pusieron en consideración elementos importantes de la crítica intempestiva, como el recuerdo, el olvido, la memoria crítica y la fuerza plástica, entre otros.

Por último y más importante, hemos analizado detalladamente la tres formas en que la historia puede servir a la vida con sus respectivas ventajas y desventajas. Así se espera que el trabajo haya resultado fecundo para una interpretación del texto nietzscheano bajo las claves de una historia monumental, anticuaria y crítica.

Podemos decir que la riqueza del planteo de Nietzsche radica particularmente en el establecimiento de la crítica de la cultura y de su sentido histórico, pero más especialmente al poner como mayor criterio de relación con la historia la vida misma como motor. Parafraseando a Espósito, podemos decir que Nietzsche nos dejó en claro que la historia puede ser algo útil y al mismo tiempo dañino (Espósito, 2006, p.149)

\section{Referencias}

Cragnolini, M. (2009). El lugar de la historia en el pensamiento de Nietzsche. En D. Brauer (Ed.), La historia desde la teoría. Volumen 2. (pp. 139-153) Buenos aires: Prometeo Libros

Correa Gutierrez, H. (2008). El sentido de la Historia en Nietzsche. La crítica al historicismo y la vida como motor de la historia. INTUS-LEGERE HISTORIA. Vol. 2, № 2, 63-72. doi: 10.15691/07176864.2008.001

Espósito, R. (2006). Bíos. Biopolítica y Filosofía. Buenos aires: Amorrortu. 
Frey, H. (2005). Nietzsche: la memoria, la historia: la Segunda intempestiva entre la crítica al historicismo y la negación de la filosofía de la historia. Reflexiones Filosóficas. 64. 271-290.

Nietzsche, F. (2002). Consideraciones Intempestivas. Buenos Aires: Alianza Editorial. 